\title{
Hepatitis B Induced Kaposi Sarcoma Flare
}

\section{Ezgi Özkur*, Mehmet Salih Gürel, Sevil Savaş, Ayşe Esra Koku Aksu and Sümeyre Seda Ertekin}

\author{
Department of Dermatology, Istanbul Training and Research Hospital, Turkey
}

*Corresponding author: Ezgi Özkur, MD, Department of Dermatology, Istanbul Training and Research Hospital, istanbul Eğitim ve Araştirma Hastanesi, Kasap Ilyas Mah, Org. Abdurrahman Nafiz Gürman Cd, 34098 Fatih/Istanbul, Turkey, Tel: +905303886781, E-mail: ezgierdal@hotmail.com

Kaposi Sarcoma (KS) is a malignant disease that originates from the vascular endothelium and has a very variable clinical course. KS is a vascular endothelial malignancy and the neoplastic cells are closely related to lymphatic endothelial cells. Although HHV-8 is found in the vast majority of all types of KS, there is no agreement on the exact mechanism of HHV-8 in KS. While immunosuppression of the host is commonly thought to promote replication of the HHV-8 virus, it is noteworthy that KS is much more strongly associated with HIV-1 infection than with immunosuppression in transplant recipients, suggesting a more complex process [1]. The four KS types (classic, HIV/AIDS-related, immunosuppression associated and African endemic) have somewhat different presentations and different clinical courses. Classic KS is usually limited the lower extremities and runs an indolent course for 10 to 15 years or more, with slow enlargement of the original tumors and the gradual development of additional lesion; whereas immunodeficiency-related diseases frequently disseminate and involve several organs.

A 53-year-old man, seen for the first time in May 2005, had a one month history of asymptomatic erythematous firm papules on his right arm and dorsal aspect of the right hand (Figure 1). There was a past history of chronic active hepatitis B infection for which the patient received lamivudine. The histopathologic examination of skin lesions showed features of Kaposi's sarcoma. Immunohistochemical study of tumor showed immune reactivity to CD-31 and HHV-8, thus a diagnosis of Kaposi's sarcoma was confirmed. Skin lesions were healed and disappeared completely within 2 weeks with topical clobetasol propionate ointment application twice daily.
In August 2014, his treatment with lamivudine changed to tenofovir with the suspicion of lamivudine resistance. Finally in September 2014, involvement of the upper eyelid, lips, face, hands and feet was noticed. On physical examination, there were disseminated multiple purplish papules and nodules on his hands, arms and feet. Also, several violaceous papules and nodules ranged from $5 \mathrm{~mm}$ to $15 \mathrm{~cm}$ were observed on the face which had started gradually over the last month (Figure 2). Routine laboratory tests were all normal. Fecal occult blood test was negative. The anti-HIV test (chemiluminescence) was negative. He had normal chest and abdominal computed tomography. Because of the aggressive course of KS, etoposide chemotherapy was started. Skin lesions diminished significantly after chemotherapy.

Kaposi's Sarcoma (KS) was first described in 1872 by Moritz Kaposi, a Hungarian dermatologist, as an "idiopathic multiple pigmented sarcoma of the skin" [2]. Kaposi's sarcoma-associated herpes virus or Human Herpesvirus 8 (HHV-8) was detected for the first time in AIDS associated KS lesions in 1994 [3].

Disseminated disease is often associated with HIV infection. Our patient was HIV negative and stated he was monogamous with marriage which lasting more than 20 years, and disavowed both homosexuality and sexual relations with commercial sex workers. He had a chronic hepatitis $B$ infection and rapid progression of the disease with involvement of labial mucosa which prompted the use of systemic chemotherapy. Xie Y, et al. reported Kaposi's Sarcoma-associated herpes virus infection rate in patients with chronic hepatitis B is significantly higher than in healthy people [4]. Although as

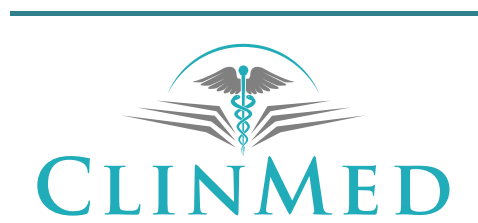

INTERNATIONAL LIBRARY
Citation: Özkur E, Gürel MS, Savaş S, Aksu AEK, Ertekin SS (2017) Hepatitis B Induced Kaposi Sarcoma Flare. Int J Virol AIDS 3:031. doi.org/10.23937/2469-567X/1510031

Received: September 10, 2016: Accepted: August 17, 2017: Published: August 19, 2017

Copyright: @ 2017 Özkur E, et al. This is an open-access article distributed under the terms of the Creative Commons Attribution License, which permits unrestricted use, distribution, and reproduction in any medium, provided the original author and source are credited. 


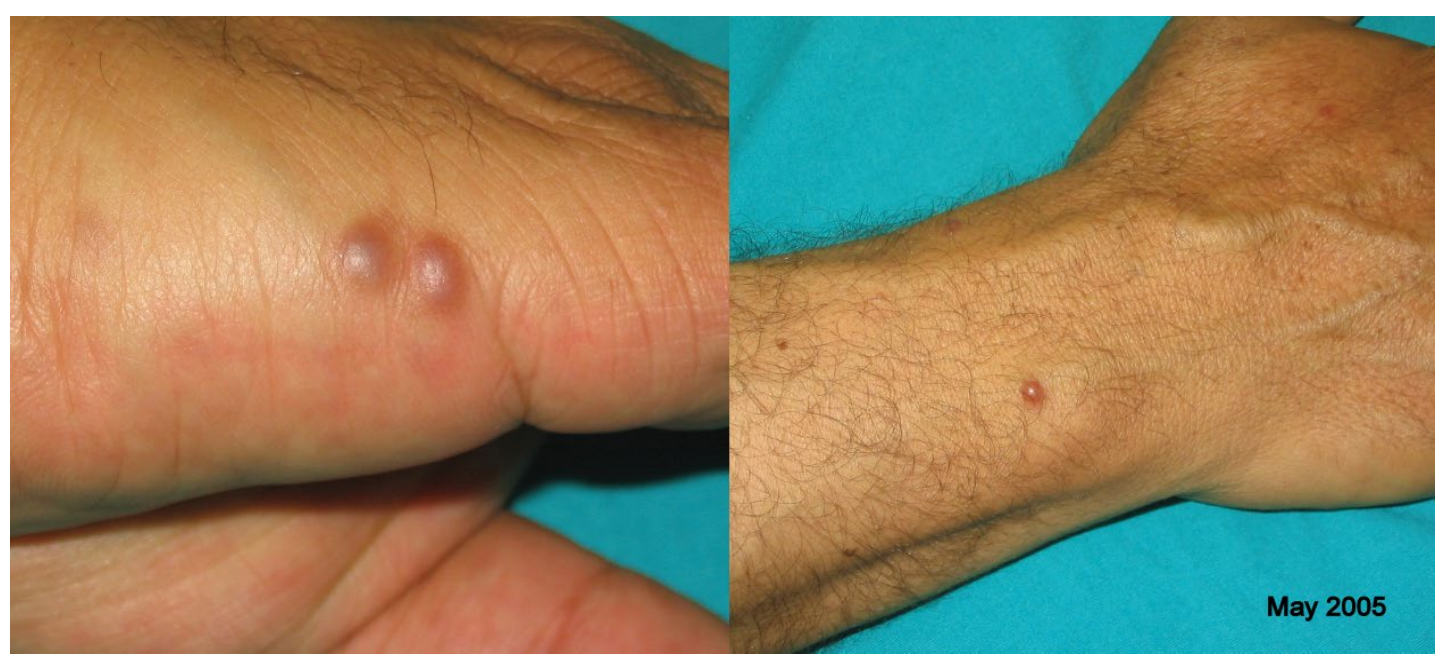

Figure 1: Erythematous firm papules on his right arm and dorsal aspect of the right hand which are less than $1 \mathrm{~cm}$ in diameter.

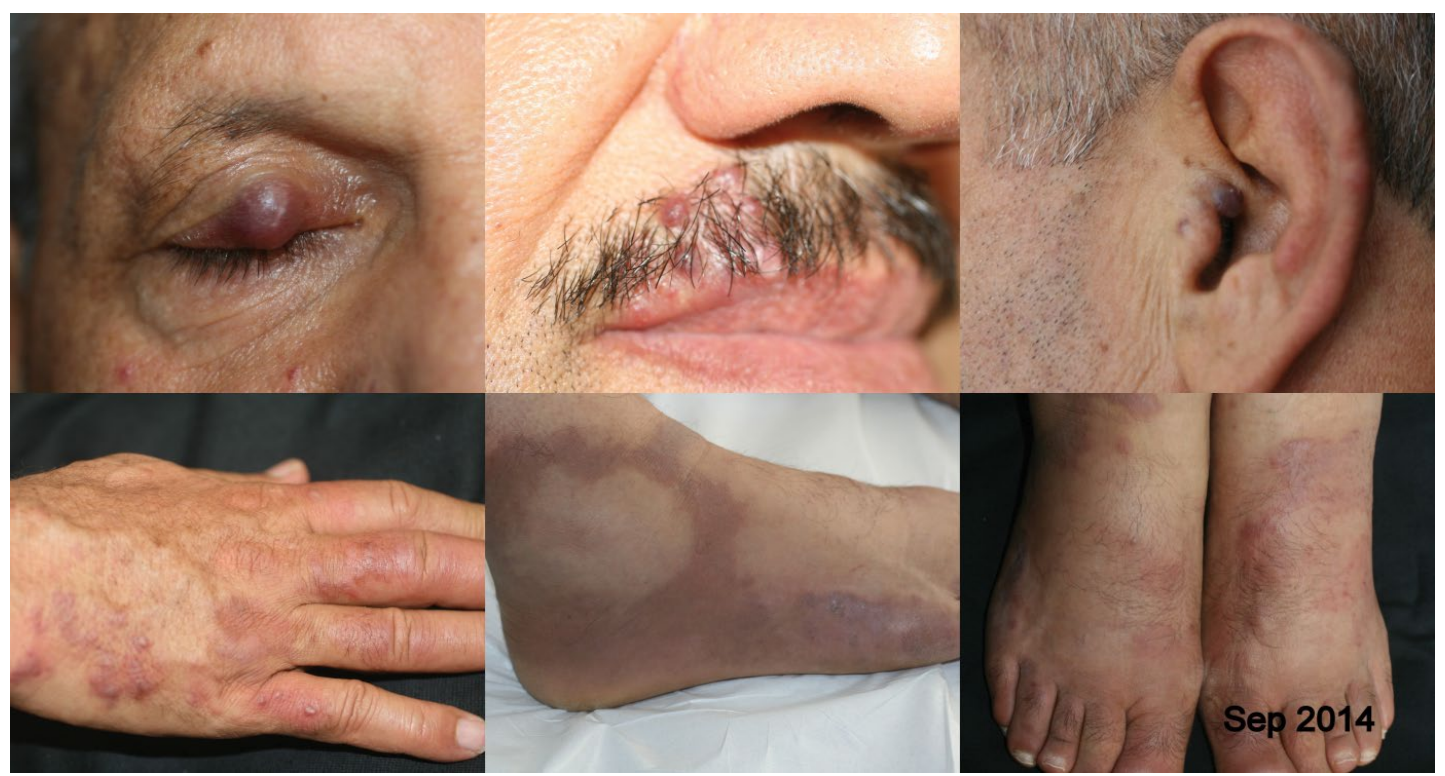

Figure 2: Disseminated multiple purplish papules and nodules on his face, hands, arms and feet which ranged from $5 \mathrm{~mm}$ to $1.5 \mathrm{~cm}$.

people age, immune senescence can cause progression of KS or it may have been the natural history of the disease but we assumed that hepatitis B can be a predisposing factor in the occurrence of Kaposi's sarcoma.

Our case report highlights the need for physicians to be aware that not only HIV infection but other chronic infections like hepatitis B constitute a risk factor for the development of disseminated KS. When the tumor does not regress or when the underlying disease is not well controlled, additional anti-tumor treatment with radiation therapy, chemotherapy, and/or surgical excision can be added.

\section{References}

1. Jean L Bolognia, Joseph L Jorizzo, Ronald P Rapini (2008) Kaposi's Sarcoma. ( $2^{\text {nd }}$ edn), Elsevier Limited, USA.

2. Moriz Kaposi (1982) Idiopathic multiple pigmented sarcoma of the skin. CA: A Cancer Journal for Clinicians 32: 342347.

3. De Vita VT HS, Rosenberg S (1997) Kaposi's sarcoma and acquired immunodeficiency syndrome. Lippincott-Raven, Philadelphia.

4. Xie Y, Ruan B, Chen Y, Wu N, Hu M, et al. (2011) Kaposi's sarcoma-associated herpesvirus infection in Chinese patients with chronic hepatitis B. J Med Virol 83: 879-883.

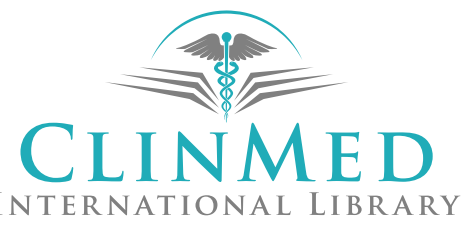

